Commun. Korean Math. Soc. 27 (2012), No. 4, pp. 835-842

http://dx.doi.org/10.4134/CKMS.2012.27.4.835

\title{
A NOTE ON THE CHARACTERIZATIONS OF PARETO DISTRIBUTION BY UPPER RECORD VALUES
}

\author{
Mohammad Ahsanullah and Mohammad Shakil
}

\begin{abstract}
Many researchers have studied the characterizations of probability distributions based on record values. It appears from literature that not much attention has been paid to the characterizations of the Pareto distribution. In this note, some new results on the characterizations of the Pareto distribution by upper record values have been established.
\end{abstract}

\section{Introduction}

Characterizations of probability distributions based on record values started in late sixties by Tata ([15]), and followed in seventies by Nagaraja ([12]) and Ahsanullah ([1], [2]). Further development continued with the contributions of Arnold et al. ([8]), Rao and Shanbhag ([14]), Ahsanullah ([1], [2], [3], [4]), Nevezorov ([13]), Lee ([11]), Bairamov et al. ([9]), Yanev et al. ([16]), Chang ([10]), Ahsanullah and Aliev ([5]), Ahsanullah and Hamedani ([6]), and Ahsanullah et al. ([7]), among others. It appears from literature that not much attention has been paid to the characterizations of the Pareto distribution. In this note, some new characterizations of the Pareto distribution based on upper record values have been given. The organization of this paper is as follows. Section 2 contains some preliminaries on record values. In Sections 3, some new results on characterizations of the Pareto distribution are established. Section 4 contains concluding remarks.

\section{Distribution of record values}

Suppose that $\left(X_{n}\right)_{n>1}$ is a sequence of independent and identically distributed (i.i.d.) random variables $\left(r v^{\prime} s\right)$ with cumulative distribution function (cdf) $F(x)$. Let $Y_{n}=\max (\min )\left\{X_{j} \mid 1 \leq j \leq n\right\}$ for $n \geq 1$. We say $X_{j}$ is an upper (lower) record value of $\left\{X_{n} \mid n \geq 1\right\}$ if $Y_{j}>(<) Y_{j-1}, j>1$. By definition $X_{1}$ is an upper as well as a lower record value. The indices at which the upper record values occur are given by the record times $\{U(n), n \geq 1\}$, where

Received October 17, 2011.

2010 Mathematics Subject Classification. 60E05, 62E10, 62E15, 62G30.

Key words and phrases. characterization, Pareto distribution, record values. 
$U(n)=\min \left\{j \mid j>U(n-1), X_{j}>X_{U(n-1)}, n>1\right\}$ and $U(1)=1$. Many properties of the upper record value sequence can be expressed in terms of the cumulative hazard rate function $R(x)=-\ln \bar{F}(x)$, where $\bar{F}(x)=1-F(x)$, $0<\bar{F}(x)<1$. If we define $F_{n}(x)$ as the $c d f$ of $X_{U(n)}$ for $n \geq 1$, then we have

$$
F_{n}(x)=\int_{-\infty}^{x} \frac{(R(u))^{n-1}}{\Gamma(n)} d F(u),-\infty<x<\infty,
$$

from which it is easy to see that

$$
F_{n}(x)=1-\bar{F}(x) \sum_{j=0}^{n-1} \frac{(R(x))^{j}}{\Gamma(j+1)}
$$

that is,

$$
\bar{F}_{n}(x)=\bar{F}(x) \sum_{j=0}^{n-1} \frac{(R(x))^{j}}{\Gamma(j+1)}
$$

If $f_{n}(x)$ is the $p d f$ of $X_{U(n)}$, then

$$
f_{n}(x)=\frac{(R(x))^{n-1}}{\Gamma(n)} f(x), \quad n \geq 1,-\infty<x<\infty .
$$

It is easy to see that $\bar{F}_{n}(x)-\bar{F}_{n-1}(x)=\bar{F}(x) \frac{f_{n}(x)}{f(x)}$. The joint $p d f$ of $X_{U(i)}$ and $X_{U(j)}$ is given by

$$
f_{i, j}\left(x_{i}, x_{j}\right)=\frac{\left(R\left(x_{i}\right)\right)^{i-1}}{\Gamma(i)} \frac{\left[R\left(x_{j}\right)-R\left(x_{i}\right)\right]^{j-i-1}}{\Gamma(j-i)} r\left(x_{i}\right) f\left(x_{j}\right)
$$

for $-\infty<x_{i}<x_{j}<\infty$. The conditional $p d f$ of $X_{U(j)} \mid X_{U(i)}=x_{i}$ is given by

$$
f\left(x_{j} \mid x_{i}\right)=\frac{f_{i j}\left(x_{i}, x_{j}\right)}{f_{i}\left(x_{i}\right)}=\frac{\left[R\left(x_{i}\right)-R\left(x_{j}\right)\right]^{j-i-1}}{\Gamma(j-i)} \cdot \frac{f\left(x_{j}\right)}{1-F\left(x_{i}\right)}
$$

for $-\infty<x_{i}<x_{j}<\infty$. For detailed treatment of the upper record values, see, for example, Ahsanullah ([4]), among others.

\section{Characterizations of the Pareto distribution}

A continuous random variable $X$ is said to have the Pareto distribution with a parameter $\beta>0$ if it has a cdf $F(x)$ of the form

$$
F(x)=1-(x)^{-\beta}, x>1, \beta>0 .
$$

In what follows, we give some new characterizations of the Pareto distribution based on upper record values. 


\subsection{A generalization of Lee's theorem}

Lee ([11]) characterized the Pareto distribution based on upper record values. In his Theorem 1 , we show that $F(x)=1-(x)^{\theta}, x>1, \theta<-1$ if and only if $(\theta+1) E\left[X_{U(n+1)} \mid X_{U(m)}=y\right]=\theta E\left[X_{U(n)} \mid X_{U(m)}=y\right], n \geq m+1$. The following theorem is a generalization of Lee's theorem.

Theorem 3.1. Suppose that $\left\{X_{n}, n \geq 1\right\}$ is a sequence of i.i.d. rv 's with absolutely continuous (with respect to Lebesgue measure) cdf $F(x)$. We assume that $F(1)=0$ and $F(x)>0, \forall x>1$. Then $F(x)=1-x^{-\delta}, \delta \geq 0, x \geq 1$ if and only if

$$
(\delta-k) E\left[X_{U(n+1)}^{k} \mid X_{U(m)}=x\right]=\delta E\left[X_{U(n)}^{k} \mid X_{U(m)}=x\right] \text { for } k<\delta .
$$

Proof. If $F(x)=1-x^{-\delta}$, then $R(x)=\delta \ln x$. It follows from (4) that

$$
\begin{aligned}
& E\left[X_{U(n+1)}^{k} \mid X_{U(m)}=x\right] \\
= & \int_{x}^{\infty} \frac{u^{k} \delta^{n-m}}{\Gamma(n-m+1)}(\ln u-\ln x)^{n-m} \frac{\delta}{u}\left(\frac{x}{u}\right)^{\delta} d u,
\end{aligned}
$$

which, on substituting $\delta \ln \frac{u}{x}=t$, easily gives

$$
E\left[X_{U(n+1)}^{k} \mid X_{U(m)}=x\right]=\left(\frac{\delta}{\delta-k}\right)^{n-m+1}
$$

Thus

$$
(\delta-k) E\left[X_{U(n+1)}^{k} \mid X_{U(m)}=x\right]=\delta E\left[X_{U(n)}^{k} \mid X_{U(m)}=x\right] .
$$

Conversely, suppose that

$$
(\delta-k) E\left[X_{U(n+1)}^{k} \mid X_{U(m)}=x\right]=\delta E\left[X_{U(n)}^{k} \mid X_{U(m)}=x\right] .
$$

Then we have

$$
\begin{aligned}
& (\delta-k) \int_{x}^{\infty} \frac{u^{k}(\ln u-\ln x)^{n-m}}{\Gamma(n-m+1)} \frac{f(u)}{\bar{F}(x)} d u \\
= & \int_{x}^{\infty} \frac{u^{k}(\ln u-\ln x)^{n-m}}{\Gamma(n-m)} \frac{f(u)}{\bar{F}(x)} d u .
\end{aligned}
$$

Cancelling $\bar{F}(x)$ from both sides of Eq.(5) and differentiating $(n-m)$ times, we obtain, on simplification, the following:

$$
\frac{f(x)}{1-F(x)}=-\frac{\delta}{x} \text {. }
$$

Integrating Eq.(6) with respect to $x$ and using the boundary conditions $F(1)=0$ and $F(\infty)=1$, we get $F(x)=1-x^{-\delta}, \delta \geq 0, x \geq 1$. This completes the proof. 


\subsection{A generalization of Chang's result}

Chang ([10]) presented some interesting results on the characterizations of the Pareto distribution based on upper record values. Unfortunately the following errors and omissions in the statements and the proof of the two theorems presented in the paper are noted.

1. Chang's Theorem 1 (Chang, [10]):

(i) $V=\frac{X_{U(n)}}{X_{U(n)}-X_{U(n+1)}}$. Since $X_{U(n+1)}>X_{U(n)}, V$ can not be greater than 1 as claimed by the author.

(ii) The marginal pdf of $V$ is given as $f_{V}(v)=\frac{\beta v^{\beta-1}}{(v-1)^{\beta+1}}, v>1, \beta>0$. It is not a pdf. For example suppose $\beta=2$, then $\int_{1}^{\infty} \frac{2 v}{(v-1)^{3}} d v=\infty$.

2. Chang's Theorem 2 (Chang, [10]):

(iii) $V=\frac{X_{U(n)}-X_{U(n+1)}}{X_{U(m)}}$. The pdf of $V$ is given as $f_{V}(v)=\frac{\beta}{(1-v)^{\beta+1}}, v<0$, $\beta>0$. It is not a pdf. For example suppose $\beta=2$, then $\int_{0}^{1} \frac{2}{(1-v)^{3}} d v=\infty$.

3. In the equation (1), (Chang, [10]), it is given $F(x)=1-\left(\frac{x}{\alpha}\right)^{-\beta}, x>1$, $\alpha>0, \beta>0$. It is not a cdf unless $\alpha=1$. We are giving the corrections.

In what follows, we will first give the corrections of Chang's results. Then a generalization of Chang's result will be given.

Theorem 3.2. Suppose that $\left\{X_{n}, n \geq 1\right\}$ is a sequence of i.i.d. rv 's with absolutely continuous (with respect to Lebesgue measure) cdf $F(x)$. Suppose $F(1)=0, F(x)>0$ for all $x>1$ and $f(x)=\frac{d}{d x} F(x)$. Then $F(x)=1-x^{-\beta}$, $x>1, \beta>0$ if and only if $\frac{X_{U(n)}}{X_{U(n+1)}-X_{U(n)}}$ and $X_{U(n)}, n \geq 1$ are independent.

Proof. If $F(x)=1-x^{-\beta}$, then the joint pdf of $X_{U(n)}$ and $X_{U(n+1)}$ is

$$
f_{U(n), U(n+1)}(x, y)=\frac{\beta^{n+1}(\ln x)^{n-1}}{\Gamma(n) x y^{\beta+1}}, 1<x<y<\infty, \beta>0 .
$$

Let $W=X_{U(n)}, V=\frac{X_{U(n)}}{X_{U(n+1)}-X_{U(n)}}$. Then

$$
X_{U(n)}=W \quad \text { and } \quad X_{U(n+1)}=\frac{V+1}{V} W .
$$

It is easy to see that the pdf of $W$ is $f_{W}(w)=\frac{\beta^{n}(\ln W)^{n-1}}{\Gamma(n) W^{\beta+1}}$. Further, we note that the Jacobian of $V$ and $W=\frac{w}{v^{2}}$. Hence, the joint pdf of $V$ and $W$ is given by

$$
\begin{aligned}
f_{V W}(v, w) & =\frac{\beta^{n+1}(\ln W)^{n-1}}{\Gamma(n) W}\left(\frac{v}{(1+V) W}\right)^{\beta+1} \frac{w}{v^{2}} \\
& =\frac{\beta^{n+1}(\ln W)^{n-1}}{\Gamma(n) W^{\beta+1}}\left(\frac{v}{1+v}\right)^{\beta+1} \frac{1}{v^{2}} .
\end{aligned}
$$

It follows from the above that $V$ is independent of $W$ and has the pdf

$$
f_{V}(v)=\beta v^{\beta-1}(1+v)^{-(\beta+1)}, v>0, \beta>0 .
$$


The joint pdf of $V$ and $W$ is

$$
f_{V W}(v w)=\frac{(R(w))^{n-1}}{\Gamma(n)} r(w) f\left(\frac{1+v}{v} w\right) \frac{w}{v^{2}} .
$$

Since $V$ and $W$ are independent, the conditional pdf of $V \mid W$ must be independent. The pdf of $W$ is

$$
f_{W}(w)=\frac{(R(w))^{n-1}}{\Gamma(n)} f(w) .
$$

Thus the pdf of $V$ is

$$
f_{V}(v)=f\left(\frac{1+v}{v} w\right) \frac{w}{v^{2}} \frac{1}{1-F(w)} .
$$

Integrating $f_{V}(v)$ from $v_{0}$ to $\infty$, we obtain

$$
1-F_{V}\left(v_{0}\right)=\left(1-F\left(\frac{1+v_{0}}{v_{0}} w\right)\right) \frac{1}{1-F(w)} .
$$

Since $F_{V}\left(v_{0}\right)$ is independent of $w$, we can write

$$
1-F\left(\frac{1+v_{0}}{v_{0}} w\right) \frac{1}{1-F(w)}=G\left(v_{0}\right) .
$$

Hence

$$
1-F\left(\frac{1+v_{0}}{v_{0}} w\right)=(1-F(w)) G\left(v_{0}\right) .
$$

Since $1 \leq w<\infty$, putting $w=1$, we get

$$
G\left(v_{0}\right)=1-F\left(\frac{1+v_{0}}{v_{0}} w\right) .
$$

Thus

$$
1-F\left(\frac{1+v_{0}}{v_{0}} w\right)=(1-F(w))\left(1-F\left(\frac{1+v_{0}}{v_{0}} w\right)\right) .
$$

Since the above equation is true for all $v_{0} \geq 0$ and all $w \geq 1$, we must have

$$
F(x)=1-x^{-\beta} \text { for all } v \geq 1 \text { and } \beta>0 .
$$

This completes the proof.

Theorem 3.3. Suppose that $\left\{X_{n}, n \geq 1\right\}$ is a sequence of i.i.d. rv 's with absolutely continuous (with respect to Lebesgue measure) cdf $F(x)$. Suppose $F(1)=0, F(x)>0$ for all $x>1$ and $f(x)=\frac{d}{d x} F(x)$. Then $F(x)=1-x^{-\beta}$, $x>1, \beta>0$ if and only if $\frac{X_{U(n+1)}-X_{U(n)}}{X_{U(n)}}$ and $X_{U(n)}, n \geq 1$ are independent.

Proof. The proof is similar to Theorem 3.2 and is omitted.

Theorem 3.4 (A generalization of Chang's result). Suppose $\left\{X_{n}, n \geq 1\right\}$ is a sequence of independent and identically distributed absolutely continuous (with respect to Lebesgue measure) random variables with cdf $F(X)$. Suppose $F(1)=$ 
$0, F(x)>0$ for all $x>1$ and $f(x)=\frac{d}{d x} F(x)$. Then $\bar{F}(x)=1-x^{-\delta}, x \geq 1$, $\delta>0$, if and only if $\frac{X_{U(m)}}{X_{U(n)}-X_{U(m)}}$ and $X_{U(m)}, 1 \leq m<n$, are independent.

Proof. We have from (4), the joint pdf of $\mathrm{X}_{U(m)}$ and $\mathrm{X}_{U(n)}$ as

$$
f_{m, n}(x, y)=\frac{(R(x))^{m-1}}{\Gamma(m)} \frac{(R(y)-R(x))^{n-m-1}}{\Gamma(n-m)} r(x) f(y)
$$

for $1 \leq m<n, x<y$.

Let $U=X_{U(m)}$ and $V=\frac{X_{U(m)}}{X_{U(n)}-X_{U(m)}}$. Then $\mathrm{X}_{U(m)}=X_{U(m)}, \mathrm{X}_{U(n)}=$ $U\left(\frac{1}{V}+1\right)$ and the Jacobian $|J|=\left|\frac{\partial\left(X_{U(m)}, X_{U(n)}\right)}{\partial(U . V)}\right|=\frac{U}{V^{2}}$. Thus the joint pdf of $U$ and $V$ is given by

$$
\begin{aligned}
& f_{U, V}(u, v) \\
= & \frac{(R(u))^{m-1}}{\Gamma(m)} \frac{\left(R\left(u\left(\frac{1}{v}+1\right)-R(u)\right)^{n-m-1}\right.}{\Gamma(n-m)} \frac{u}{v^{2}} r(u) f\left(u\left(\frac{1}{v}+1\right)\right)
\end{aligned}
$$

for $1 \leq u<\infty, v \geq 0$. Substituting $R(x)=\delta \ln x, f(x)=\delta x^{-(\delta+1)}$, we obtain from $(8)$

$$
\begin{aligned}
& f_{U, V}(u, v) \\
= & \frac{\delta^{m-1}(\ln x)^{m-1}}{\Gamma(m)} \frac{\left(\delta \ln \left(\frac{1}{v}+1\right)^{n-m-1}\right.}{\Gamma(n-m)} \frac{\delta}{x} \frac{u}{v^{2}} \delta\left[u\left(\frac{1}{v}+1\right)\right]^{-(\delta+1)}, 1 \leq u<\infty, v \geq 0 .
\end{aligned}
$$

Thus $U$ and $V$ are independent. Suppose $U=X_{U(m)}$ and $V=\frac{X_{U(m)}}{X_{U(n)}-X_{U(m)}}$. Then from (8), we must have the conditional pdf of $V \mid U=u$ as

$$
f_{V \mid, U}(v \mid u)=\frac{\left(R\left(u\left(\frac{1}{v}+1\right)-R(u)\right)^{n-m-1}\right.}{\Gamma(n-m)} \frac{f\left(u\left(\frac{1}{v}+1\right)\right)}{\bar{F}(u)},
$$

which will be independent of $u$. We can write (9) as

$$
f_{V, \mid U}(v \mid u)=\frac{\left(R\left(\frac{1}{v}+1\right)\right)^{n-m-1}}{\Gamma(n-m)} \frac{u}{v^{2}} \frac{f\left(u\left(\frac{1}{v}+1\right)\right)}{\bar{F}(u)}
$$

for $1 \leq u<\infty, v \geq 0$. Since (10) is independent of $U$, we must have

$$
\frac{1}{v^{2}} \frac{f\left(u\left(\frac{1}{v}+1\right)\right)}{\bar{F}(u)} \frac{f\left(u\left(\frac{1}{v}+1\right)\right)}{\bar{F}(u)},
$$

independent of $u$ for all $1 \leq u<\infty, v \geq 0$.

Suppose $\frac{1}{v^{2}} \frac{f\left(u\left(\frac{1}{v}+1\right)\right)}{\bar{F}(u)}=g(v)$, where $g(v)$ is independent of $u$. Taking $u \rightarrow 1$, we obtain $g(v)=\frac{1}{v^{2}} f\left(\frac{1}{v}+1\right)$. Thus

$$
f\left(u\left(\frac{1}{v}+1\right)\right)=f\left(\frac{1}{v}+1\right) \bar{F}(u)
$$


for all $1 \leq u<\infty, v \geq 0$. Substituting $t=\frac{1}{v}+1$ in (12) and integrating both sides of (12) with respect to $t$ from $t_{0}$ to $\infty$, we obtain

$$
\bar{F}\left(t_{0} u\right)=\bar{F}\left(t_{0}\right) \bar{F}(u)
$$

for all $u, 1 \leq u<\infty$ and $t_{0} \geq 1$. The solution of Eq.(13) with the boundary conditions $\bar{F}(1)=1$ and $\bar{F}(\infty)=0$ is

$$
\bar{F}(x)=1-x^{-\delta}, x \geq 1, \delta>0 .
$$

This completes the proof.

\section{Concluding remarks}

In this paper, some results on characterizations of Pareto distribution based on upper record values have been obtained. It is hoped that the findings of this paper will be useful for the practitioners in various fields of studies and further enhancement of research in the fields of distribution theory and record values, and their applications.

\section{References}

[1] M. Ahsanullah, Record values and the exponential distribution, Ann. Inst. Statist. Math. 30A (1978), no. 3, 429-433.

[2] Characterization of the exponential distribution by record values, Sankhya Ser. B 41 (1979), no. 1-2, 116-121.

[3] - Some characteristic properties of the record values from the exponential distribution, Sankhya Ser. B 53 (1991), no. 3, 403-408.

[4] _ Record Values-Theory and Applications, University Press of America, Lanham, $\mathrm{MD}, 2004$.

[5] M. Ahsanullah and F. Aliev, Some characterizations of exponential distribution by record values, J. Statist. Res. 42 (2008), no. 2, 41-46.

[6] M. Ahsanullah and G. G. Hamedani, Exponential Distribution-Theory and Methods, Nova Science Publications, New York, 2010.

[7] M. Ahsanullah, G. G. Hamedani, and M. Shakil, On record values of univariate exponential distribution, Journal of Statistical Research 44 (2010), no. 2, 267-288.

[8] B. C. Arnold, N. Balakrishnan, and H. N. Nagaraja, Records, John Wiley \& Sons Inc., New York, 1998.

[9] I. G. Bairamov, M. Ahsanullah, and A. G. Pakes, A characterization of continuous distributions via regression on pairs of record values, Aust. N. Z. J. Stat. 47 (2005), no. $4,543-547$.

[10] S.-K. Chang, Characterizations of the Pareto distribution by the independence of record values, Journal of the Chungcheong Mathematical Society 20 (2007), no. 1, 51-57.

[11] M.-Y. Lee, Characterizations of the Pareto distribution by conditional expectations of record values, Commun. Korean Math. Soc. 18 (2003), no. 1, 127-131.

[12] H. N. Nagaraja, On a characterization based on record values, Austral. J. Statist. 19 (1977), no. 1, 70-73.

[13] V. B. Nevzorov, Records: Mathematical Theory, American Mathematical Society, Providence, Rhode Island, 2000. 
[14] C. R. Rao and D. N. Shanbhag, Recent approaches for characterizations based on order statistics and record values, Handbook of Statistics, (N. Balakrishnan and C. R. Rao eds.), 10 (1998), 231-257.

[15] M. N. Tata, On outstanding values in a sequence of random variables, Z. Wahrscheinlichkeitstheorie und Verw. Gebiete 12 (1969), 9-20.

[16] G. Yanev, M. Ahsanullah, and M. I. Beg, Characterizations of probability distributions via bivariate regression of record values, Metrika 68 (2008), no. 1, 51-64.

Mohammad Ahsanullah

Department of Management SCIEnCES

RIDER UNIVERSITY

LAWRENCEVILLE, NJ 08648, USA

E-mail address: ahsan@rider.edu

MOHAMmad SHAKIL

Department of Mathematics

Miami Dade College

Hialeah Campus Hialeah, Fl 33012, USA

E-mail address: mshakil@mdc.edu 